\title{
Improved method for Mica functionalization used in single molecule imaging of DNA with atomic force microscopy
}

\author{
Hana Zapletalová ${ }^{1, *}$, Jan Přibyl ${ }^{2}$, Marcel Ambrož ${ }^{2}$, Milan Vůjtek ${ }^{3}$, Petr Skládal ${ }^{2}$, Hana Kolářová ${ }^{1}$ \\ ${ }^{1}$ Department of Medical Biophysics and Institute of Molecular and Translation Medicine, Faculty of Medicine \\ and Dentistry, Palacký University Olomouc, Hněvotínská 3, CZ-77515 Olomouc, Czech Republic \\ ${ }^{2}$ Central European Institute of Technology and Department of Biochemistry, \\ Masaryk University, Kamenice 5, CZ-62500 Brno, Czech Republic \\ ${ }^{3}$ Department of Experimental Physics, Faculty of Science, \\ 17. Listopadu 1192/12 Palacký University,CZ-771 46 Olomouc, Czech Republic
}

\begin{abstract}
The modified procedure of 1-(3-aminopropyl)silatrane (APS) compound synthesis based on a new derivative (3-aminopropyl)trimethoxysilane for the purpose of DNA immobilization for AFM single imaging is described. New reaction pathway based on kinetically driven reaction approach is described. Necessity of two-step purification process is proved; ability of purified APS to provide four times smoother surfaces in comparison with a crude product is demonstrated. Various analytical methods such mass spectroscopy and ${ }^{1} \mathrm{H}$ NMR were used to show structure and enhanced purity of the APS product. APS mediates fixation of DNA molecules to mica substrates to be used for DNA imaging under Atomic Force Microscope. The use of an APS compound for simple and rapid silanization of mica surface is demonstrated. The advantages of APS-based method are based mainly on low roughness of modified mica and homogeneous surface coverage by short sequence dsDNA (246 bp). The product obtained by the condensation reaction was purified in a two step process whose effectiveness was demonstrated not only by reduction of the silanized surface roughness, but also by mass spectroscopy (MS-ESi), MALDI-TOF method and proton magnetic resonance spectroscopy. Experiments demonstrate that 1-(3-aminopropyl)silatrane can be used to fix dsDNA molecules to a mica surface to be visualized by either the tapping mode or the force-volume mode of AFM microscopy, as demonstrated by experiments. Moreover, necessity of advanced purification protocol is demonstrated by AFM based roughness measurements - pure vs crude APS product. The kinetics of APS-layer aging, caused by silicon oxide growth on silanized layers, was studied by water contact angle measurements and is discussed.
\end{abstract}

Keywords: AFM (atomic force microscopy); Surface modification; Surface chemistry; APS synthesis; DNA immobilization.

\section{Introduction}

Molecular biological methods dealing with DNA molecules have undergone an extreme development and expansion during last decades. However, there is not any commercially available method that allows for direct visualization of DNA molecules. This opportunity for this direct visualization is brought by Atomic Force Microscopy (AFM) ${ }^{1-3}$, a part of scanning probe microscopy (SPM). SPM methods are based on interaction between the sharp probe and surface. The surface structure (or other surfaces properties) is afterwards reconstructed based on specific signal (force, current, charge) measured between the probe tip and the surface area, above which the probe is rasterized. Moreover, AFM microscopy brings the possibility to visualize the

*Corresponding author: Hana Zapletalova

Email address: hana.zapletalova@gmail.com

DOI : http://dx.doi.org/10.13171/mjc55/01607041620/zapletalova
DNA molecules in a dry state, but also in liquid, i.e. in the natural environment, such as in buffer solution. DNA molecules to be imaged by AFM need to be properly immobilized on atomically smooth surfaces presented by muscovite mica in most cases. Proper immobilization involves stable fixation of DNA molecules with optimal surface density. Various immobilization procedures were tested for this purpose to, as it will be discussed below ${ }^{4-10}$. DNA immobilization process was also used in numerous studies of DNA mechanical ${ }^{11}$ and binding properties ${ }^{12}$, where high resolution of AFM microscope was used.

As will be discussed in this paper, APS (aminopropylsilatrane) method seems to be the most promising on the way to ideal one - purpose highly-ordered pyrolytic graphite (HOPG) and

Received May $14^{\text {th }}, 2016$ Accepted June $8^{\text {th }}, 2016$ Published July $4^{\text {th }}, 2016$ 
muscovite (mica). HOPG materials adsorb biomolecules non-specifically by strong hydrophobic interaction, which can lead to denaturation of such molecules. Therefore, the mica substrate is usually used for this kind of visualization. From a chemical point of view, it is a phyllosilicate mineral, negatively charged on its surface, usually described as hydrated silicon oxide with a $p K a$ value around $3.0^{13}$. This material shows hydrophilic properties (water contact angle below $5^{\circ}$ ) and can be easily modified in many chemical ways. The use of buffer with $\mathrm{pH}$ lying between $\mathrm{pKa}$ values of mica and protein is a simple and effective method for protein immobilization onto the mica surface ${ }^{14}$. The presence of positive charge protein molecules above the negatively charged mica surface leads to charge-based protein immobilization, providing protein fixation stability even in the liquid environment. On the contrary, a DNA molecules due to the presence of sugar-phosphate chain have a $p K a$ value close to the same value of mica material. The existence of negative charges on both entities complicates the practical performance of the immobilization method ${ }^{15}$.

In general, two modifications of the bare mica surface are used for DNA immobilization. In one case, DNA is immobilized on mica by deposition from a solution containing divalent ions ${ }^{16}$. It is well known that divalent ions act as $\mathrm{Mg}^{2+}$ bridges

between DNA and the mica substrates, leading to a weak adsorption of DNA. The use of magnesium ions provides fixation not stable enough to visualize DNA under liquid conditions. The use of heavy metal divalent cations $\left(\mathrm{Co}^{2+}, \mathrm{Ni}^{2+}\right)$ leads not only to bridges between DNA and the mica, stable under liquid environment, however also to DNA self-crosslinking. A second modification of mica consists of treating the mica surface with 3-aminopropyltriethoxysilane(AP-mica) ${ }^{17,18}$ (Figure 1), leading to a strong adsorption of the DNA molecules ${ }^{6}$. However, APTES compound is very sensitive to the presence of water, even water vapors cause the formation of matrix structures, disruptive to the AFM imaging ${ }^{19}$.

The elimination of aminopropylsilanes polymerization is a necessary condition to be used as a mediating layer for AFM imaging by reason of atomically flat surface. It is difficult to eliminate the adsorption of trace quantities of water from nonaqueous solvents ${ }^{20}$ on the other hand adsorbed water is easily removed from fumed silica by gasphase evacuation at room temperature ${ }^{21}$. The use of a gas-phase process also allows for much higher reaction temperatures than can be tolerated in solution reactions. Thus water is not needed at high reaction temperatures. A direct reaction of gaseous alkoxy- or chlorosilanes with surface hydroxyl groups on silica occurs at $300{ }^{\circ} \mathrm{C}^{22,23}$.
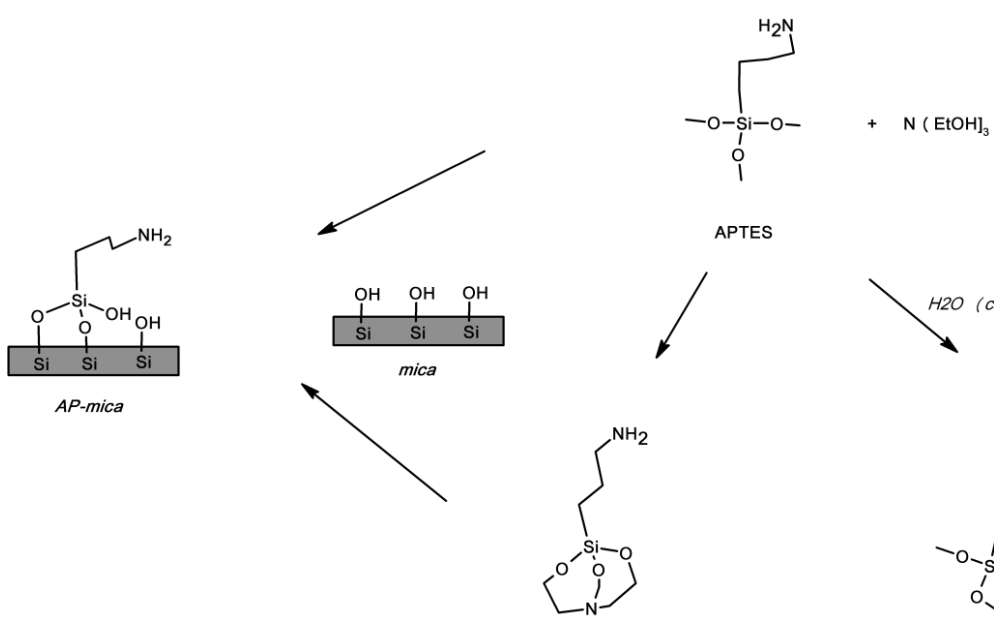

APS
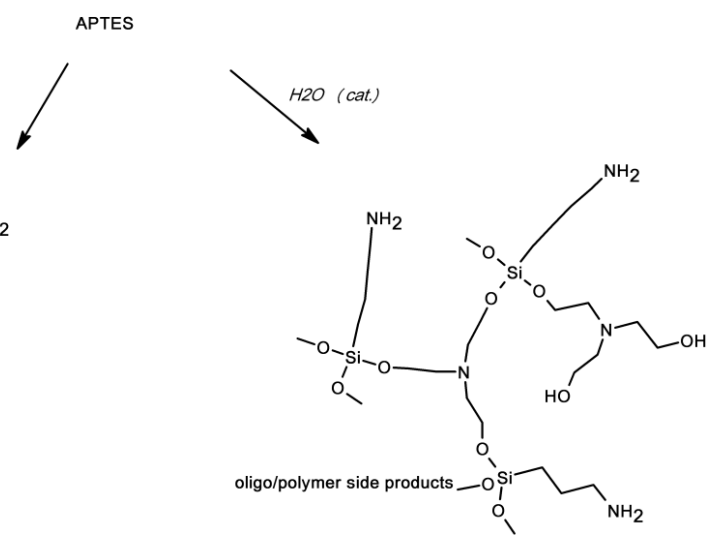

Figure 1. APS compound is formed during reaction of (3-aminopropyl)trimethoxysilane (APTMS) with triethanolamine (TEOA) as shown in the upper reaction. The lower reaction scheme shows formation of possible reaction side products.

Another polymer-free approach in preparation of silanized layers is the use of monoalkoxysilane (3-aminopropyl)dimethylethoxysilane (APDMES). Based on its structure, APDMES cannot create polymeric structures, it can either modify the surface or form a dimer, thus not affecting the AFM imaging of small biomolecules immobilized through its moiety. Moreover, APDMES has sufficient vapor pressure for gas-phase adsorption studies ${ }^{19}$.

Aminopropyl silatrane (APS) was found ${ }^{24-26}$ to be a convenient replacement for either APTES and APDMES in an attempt to avoid their undesirable properties. The use of APS allows for a one step silanization of surfaces (i.e. mica) with an aqueous, long-term stable solution. DNA molecules are 
adsorbed during the following step and can be visualized afterwards by AFM either in air as well as in aqueous solutions. As APS is not commercially available APS preparation proceeds with a single step condensation reaction between the APTES and triethanolamine with catalytical amount of metal sodium (Figure 1). Purification of the final product is presented by vacuum distillation, removing the unreacted APTES molecules.

In this paper an improved procedure for the preparation of aminopropyl silatrane compound including the reasons for the improving of the preparation procedure, such as MALDi-TOF mass spectrometry, AFM roughness analysis etc is presented. The effect of reaction ratio, time, temperature as well as the role of metal sodium catalysis in the synthesis of APS compound is shown and discussed. The APTES used in other study ${ }^{27}$ was replaced by (3-Aminopropyl)trimethoxysilane representing structurally similar but more reactive compound. Moreover, the necessity of purification and recrystallization steps is revealed. To support our claims the roughness analysis and AFM imaging of APS-mediated immobilization of dsDNA of $246 \mathrm{bp}$ length is presented in this paper.

\section{Experimental Section}

\section{Reagents and Material}

All commercially available reagents used were of the highest purity grade. (3aminopropyl)trimethoxysilane (APTMS), 3aminopropyldimethylethoxysilane (APDMES) triethanolamine (HOCH2CH2)3N (TEOA), diisopropylamine (DiPEA), HEPES and $\mathrm{Na}$ were purchased from Sigma-Aldrich and used as received, without further purification. Methanol $(\mathrm{MeOH})$, toluen, tetrahydrofuran (THF) was purchased from LachNer.Mica strips were purchased from TedPella (USA), grade V1. 246 bp dsDNA (double stranded) fragments used for AFM imaging were products of standard PCR multiplication protocol, purified using Sephadex column.

\section{APS Synthesis}

1-(3-aminopropyl)silatrane (APS) is not a commercially available compound. The synthesis of this reagent was described in a limited number of the publications ${ }^{26,27}$. The basic steps of APS synthesis were adopted from the previous publication ${ }^{27}$. However, the procedure was modified in order to improve yield and to decrease amount of side-products of the synthesis. The two opposite ways of synthesis were chosen to be tested.

First, the thermodynamic approach to reaction driving was tested, An equimolar amount $(10 \mathrm{mmol})$ of APTMS and TEOA compound was left to react with a catalytical amount of metal sodium $(1 \mathrm{mmol})$ in $100 \mathrm{~mL}$ of anhydrous THF. For more details on the reaction procedure (temperature, reaction time) see Table 1. The purpose of this way of synthesis was to separate the reacting molecules and thus suppress the side reaction products.

The kinetic driving of synthetic reaction was chosen as the second approach to the synthesis. Various ratios of compounds, reaction times and the catalytical effect of metal sodium were tested, for more details on the reaction procedure see Table 1 . The typical amount of APTMS in the reaction was $10 \mathrm{mmol}$, the weight of sodium used as catalyzer was $1 \mathrm{mmol}$, when this kind of catalysis was included. As APTMS and TEOA are liquid under room temperature, these chemicals were mixed to give a reaction mixture with no solvent added. Products of both reaction types (kinetic and thermodynamic) were purified before further use for analysis and surface modification, as described in the next chapter.

The experimental section should contain information on materials, methods, analytical instruments used, spectral data and all necessary informations to help readers to understand the experiences etc.

\section{APS Purification}

APTMS (bp 91-92 ${ }^{\circ} \mathrm{C} / 15 \mathrm{mmHg}$ ) and TEOA (bp $190-193{ }^{\circ} \mathrm{C} / 5 \mathrm{mmHg}$ ) were removed from the crude reaction mixture by vacuum evaporation $(0.08 \mathrm{mmHg})$. The polymeric side products were removed by toluene extraction of APS, followed by toluene recrystallization.

\section{MS-ESi (MS/MS) of APS}

Samples were measured by direct infusion into an MS spectrometer via a builted-in syringe pump, flow rate $5 \mu \mathrm{L} / \mathrm{min}$. Samples of APS obtained by toluene recrystalization were dissolved in water and proceeded to MS-ESi (MS/MS) detection.

An Orbitrap Velos Pro MS spectrometer (ThermoFisher Scientific, Bremen, Germany) equipped with an HESI II probe was used for determination of APS sample composition according to molecular mass content. The following settings of the HESI Probe were used for all experiments: positive mode, heater temperature $50{ }^{\circ} \mathrm{C}$, sheath gas flow rate $10 \mathrm{arb}$, aux gas flow rate $3 \mathrm{arb}$, sweep gas flow rate $0 \mathrm{arb}$, ispray voltage $4 \mathrm{kV}$, capillary temperature $250{ }^{\circ} \mathrm{C}$, s-lens RF level $55 \%$. Compound detection: HRMS mode, resolution $30000, \mathrm{~m} / \mathrm{z}$ range 100-1000, MS/MS fragmentation, parent ion $\mathrm{m} / \mathrm{z} 233$, collision energy $40 \%, \mathrm{~m} / \mathrm{z}$ range $60-300$. MS3 fragmentation: parent ion $\mathrm{m} / \mathrm{z} 233174$, collision energy $40 \%, \mathrm{~m} / \mathrm{z}$ range 50-300.

\section{MALDi-TOF of APS}

The sample of APS solutions in toluene (used for recrystalization, as mentioned in section 2.3) in a volume of $0.6 \mu \mathrm{L}$ was mixed with $2.4 \mu \mathrm{L}$ of the MALDi matrix solution $(20 \mathrm{mg} / \mathrm{mL})$ 2,5-dihydroxybenzoic acid in a mixture of acetonitrile and water, $1: 1, \mathrm{v} / \mathrm{v})$ and $0.6 \mu \mathrm{L}$ of this 
mixture were spotted on a stainless steel MALDi target and dried at room temperature. MALDi-TOF MS measurements were carried out using an Ultraflex III instrument (Bruker Daltonik, Bremen, Germany) operated in the reflectron positive ion mode.

\section{Immobilization of DNA onto the Mica Surface}

Mica (grade V-1 highest possible quality, TedPella, USA) was always cleaned immediately prior the further modification by subsequent removal of three layers with a standard scotch tape. Cleaning of the surface was finished by compressed air pressure which removed the remaining parts of the mica.

\section{APDMES protocol}

An APDMES protocol for vapour silanization of mica surfaces ${ }^{28}$ combines advantages of the APTES protocol ${ }^{17}$ leading often to generation of nanometer size objects on mica surface, with commercially available monoalkoxy-derivative of siloxane. Freshly cleaned mica strips were placed inside the $30 \mathrm{~cm}$ desiccator above the layer of drying agent (silica gel) together with two small Eppendorf tubes $(0.5 \mathrm{~mL})$ containing $50 \mu \mathrm{L}$ of DiPEA as a catalyzer and $50 \mu \mathrm{L}$ of APDMES. The desiccator was evacuated for 10 minutes by oil vacuum pump (10 $\mathrm{Pa}$ value of vacuum), then the main valve was closed and the procedure runs for following 80 minutes under the ambient temperature.

Deposition of DNA molecules onto the AP-mica surface was done as follows: $5 \mu \mathrm{L}$ of $1 \mu \mathrm{g} / \mathrm{mL}$ DNA solution in $40 \mathrm{mM}$ HEPES buffer $\mathrm{pH} 7.45$ was pipette in the center of AP-mica slide. After 15 minutes incubation at $5^{\circ} \mathrm{C}$, when the mica sample was placed inside standard $30 \mathrm{~mm}$ plastic Petri dish, unbound amount of DNA was washed out from the mica surface by large amount of double distilled water.

Samples of DNA immobilized on the mica surface were kept in the desiccator above the layer of drying agent prior the AFM visualization.

\section{APS protocol}

The protocol for APS modification of the mica surface was adopted from the previous publication ${ }^{\mathbf{2 6}, 29}$. Briefly, $15 \mu \mathrm{L}$ of 3000 -times diluted APS stock solution $(10 \mathrm{mg} / \mathrm{mL})$ in double distilled water was placed in the center of a freshly cleaved mica slide. After 20 minutes of incubation under ambient conditions, the excess APS was removed by a large amount of double distilled water and the surface was dried by compressed air stream.

The APS-mica surface was used either for DNA immobilization using the method mentioned above or for contact angle and roughness experiments.

\section{AFM imaging of immobilized DNA}

A Bruker FastScan AFM instrument (Bruker Nano, Santa Barbara, CA, USA) was used for imaging all the samples. To visualize the sample surface, a Scan Assist mode with ScanAssyst probes containing a silicon tip on a silicon nitride cantilever (Bruker Nano, Santa Barbara, CA, USA) with a sty76iffness of $k=18 \mathrm{~N} / \mathrm{m}$ and a resonant frequency $f=1400 \mathrm{kHz}$ were used. The sample to be studied by AFM microscopy was fixed to the instrument base by a vacuum, as recommended by the microscope producer. Following parameters were found to be optimal for either silanized only or DNA-containing surface visualization by the ScanAssyst mode of the Bruker FastScan microscope: maximal Z-scale range $350 \mathrm{~nm}$ (to be driven by feedback loop), scan rate of $2 \mathrm{~Hz}$, resolution of the AFM images was set to be $1024 \times 1024$, feedback loop intensity varied from 0.1 up to 0.2 , depending on the sample surface properties.

Ten AFM scans over randomly chosen areas of modified mica surface were recorded for each surface modification. At least 5 images of this series were giving similar results - the parameter of significance was either DNA presence or surface roughness or both parameters. The AFM image lying in the middle of this significance interval was afterwards chosen for publication.

Rough images captured by AFM microscope were processed afterwards by Gwyddion software ver. 2.24 (free software under GNU General Public License ${ }^{30}$. The main steps used here were: leveling, line adjustment according to median, Z-scale coloration and export to a PNG image file format.

\section{Roughness analysis}

Roughness of studied surfaces was characterized by following parameters - $R_{a}$ (average roughness, Eq. 1) and $R_{m s}$ (root mean square roughness, Eq. 2)

Average roughness is defined as the average deviation of all points roughness profile from a mean line over the evaluation length:

$$
\boldsymbol{R}_{a}=\frac{1}{N} \sum_{j=1}^{N}\left|r_{j}\right| \quad \text { Eq. } 1
$$

The average of the measured height deviations taken within the evaluation length and measured from the mean line:

$$
\boldsymbol{R}_{m s}=\sqrt{\frac{1}{N} \sum_{j=1}^{N} r_{j}^{2}} \quad \text { Eq. } 2
$$

Gwyddion software was used to calculate the roughness of various samples, when the Statistical Quantities module was employed. Identical area size $\left(1 \mu \mathrm{m}^{2}\right)$ of each sample was processes to obtain comparable results. 


\section{Contact Angle Measurement}

See System instrument (AdveX instruments, Brno, Czech Republic) containing 3-axis positioning table with HD camera connected to PC via standard USB cable was used for contact angle measurements. Only the static water contact angle was captured, when $5 \mu \mathrm{L}$ of double distilled water (resistance $\leq$ $0.01 \mathrm{M}$ Siemens) was dropped from a Gilson Microman CP M10 pipette (Gilson, Villiers le Bel, France) for highly viscous solutions, kept always in $90^{\circ}$ angle to surface, $4 \mathrm{~mm}$ above it.

The image of the droplet was taken by the HD camera, as a part of the See System instrument for contact angle measurement and surface energy determination (AdveX instruments, Brno, Czech Republic). To take the image of the droplet the constant span of $5 \mathrm{~s}$ after the drop was deposited on the sample was kept in all the cases. The reported value is an average of the results obtained on three samples of ten droplets each of studied surface, evaluated afterwards by the See System software by method of 3 point interpolation.

The aging of the silanized layers was determined as the change of contact angle, measured as described above, in following time intervals: $0.5,3$, 18,24 and 48 hours. The averaged values of water contact angles were plotted against the time scale and fitted with linear regression analysis to show the process of layer aging. APS-modified mica was kept in the evacuated desiccator during the time periods between the measurements.

\section{Results and Discussion \\ APS synthesis}

Various batches of APS were prepared by condensation reaction between the APTMS and TEOA under various experimental conditions, as summarized in the Table 1. First, the thermodynamic and kinetic way of reaction driving was tested, together with influence of metal sodium catalyst on reaction yield and overall process.

Reaction thermodynamics involves the effect of temperature on the overall course of the reaction, i.e. the origin of individual products, their presence in the final mixture of reaction products and yield of the reaction. The low temperature should support slow reaction way leading to the reaction ratio $1: 1$ was used in all of the case, as well as sodium catalyst, the influence of reaction temperature was studied. It was found, only the reaction product led to the APS product, however of very small yield and quality.

Reactions of APS with triethanolamine labeled with the K-prefix (Table 1) have led to colorless crystalline products of reaction yields that vary between $42 \%$ and $65 \%$. Higher yield values were obtained for the reactions catalyzed by metal sodium.

Reaction labeled as A was performed as an identical copy of the reaction process published by Dumitriu et al. ${ }^{27}$. A crystalline product of reaction yield $81 \%$ was further inspected by other methods, as described in the chapters below.

Table 1. Composition of various synthetic ways of APS preparation.

\begin{tabular}{|c|c|c|c|c|}
\hline Product label & $\mathrm{T}\left[{ }^{\circ} \mathrm{C}\right]$ & Reaction ratio* & $\mathrm{Na}(\text { cat. })^{* * *}$ & Reaction time [h] \\
\hline T01 & 40 & $1: 1$ & YES & 48 \\
\hline T02 & RT & $1: 1$ & YES & 1 week \\
\hline T03 & 60 & $1: 1$ & YES & 4 \\
\hline K01A & 80 & $1: 1.5$ & YES & 2 \\
\hline K01B & 80 & $1: 1$ & YES & 2 \\
\hline K01C & 80 & $1.5: 1$ & YES & 2 \\
\hline K02A & 80 & $1: 1.5$ & NO & 4 \\
\hline K02B & 80 & $1: 1$ & NO & 4 \\
\hline K02C & 80 & $1.5: 1$ & NO & 4 \\
\hline K03A & 80 & $1: 1.5$ & YES & 6 \\
\hline K03B & 80 & $1: 1$ & YES & 6 \\
\hline K03C & 80 & $1.5: 1$ & YES & 6 \\
\hline K04A & 80 & $1: 1.5$ & NO & 6 \\
\hline K04B & 80 & $1: 1$ & NO & 6 \\
\hline K04C & 80 & $1.5: 1$ & NO & 6 \\
\hline $\mathbf{A}^{\#}$ & 60 & $1: 1$ & YES & 4 \\
\hline
\end{tabular}

$\mathrm{K}$ - prefix - reaction procedure driven by kinetics, $\mathrm{T}$ - prefix - - reaction procedure driven by thermodynamics * triethanolamine: aminopropyltrimethoxysilane molar ratio, ** presence of sodium metal as catalyst in reaction,

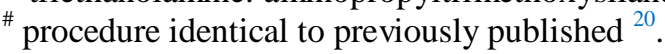

\section{APS purification}

The crude reaction products were found to contain mainly oligo and polymeric byproducts, increasing rapidly the surface roughness of APS-modified mica surface. Therefore we applied another purification step beside the vacuum evaporation of unreacted APTMS. Chromatographic methods are very effective methods for purification of the chemical compounds; however most of the stationary phases use silica base materials, easily modifiable by APS/APTES compounds. Therefore we employed recrystallization with a hot toluene thus producing highly purified APS, as was characterized by MALDi-TOF method. However, it is a quite time consuming method, needs some time 
interval before the final product is ready for final use.

\section{MS-ESi (MS/MS) of APS}

Electrospray mass spectrometry is a useful tool to determine the molecular weight of substances represented by the reaction product. The MS-ESi method was used in this study to prove whether or not after purification the expected molecule (APS) is present in the final product of the reaction. Presence of the APS compound in the mass spectra (see an example of spectra T03A in the supplementary data of this article) is proven by the presence of the main molecular peaks $\mathrm{m} / \mathrm{z}$ value of 231.5 , the closest value to the basic molecular weight of the non-ionized APS molecule (232.1). There were also found other molecular ion (m/z 174.05 and 192.1) of APS compound formed by post-ionization process.

The MS-ESi method has only semi-quantitative informational value indicating that molecular peak intensity is not directly proportional to the compound content in the mixture. However, this method can be used to determine whether a molecule is or is not represented in the mixture. Neither the basic molecular ion of TEOA (Mw 149.1) nor of APTMS (Mw 179.29) can be found in the mass spectrum of the APS product labeled as T03A, measured after toluene recrystallization (Figure 2).

Due to the APS tendency to modify any silicate surface, it is very difficult to analyze byproducts by traditional analytical and/or structural methods.

\section{${ }^{1} \mathrm{H}$ NMR}

$1 \mathrm{H}$ NMR spectra of APS was acquired in $\mathrm{D}_{2} \mathrm{O}$ with $500 \mathrm{MHz}$ NMR spectrometer Bruker equipped with $3 \mathrm{RF}$ channels and $5 \mathrm{~mm}$ nitrogen-cooled dual (BB-1H) cryoprobe (Prodigy).

Following chemical shifts $\delta$ were found in the proton NMR spectra of purified APS compound $\left(500.25 \mathrm{MHz}, \mathrm{D}_{2} \mathrm{O}\right): 1.066-1.068(2 \mathrm{H},-\mathrm{Si}-\mathrm{CH} 2-)$, 1.744-1.746 (2H, Si-CH2- $\mathrm{CH} 2-\mathrm{CH} 2-\mathrm{NH} 2)$; 2.593-2.595 (2H, Si-CH2-CH2-CH2-NH2); 3.1383.139 (6H, N-CH2-CH2-O-); 3.797-3.798 (6H, N$\mathrm{CH} 2-\mathrm{CH} 2-\mathrm{O}-)$.

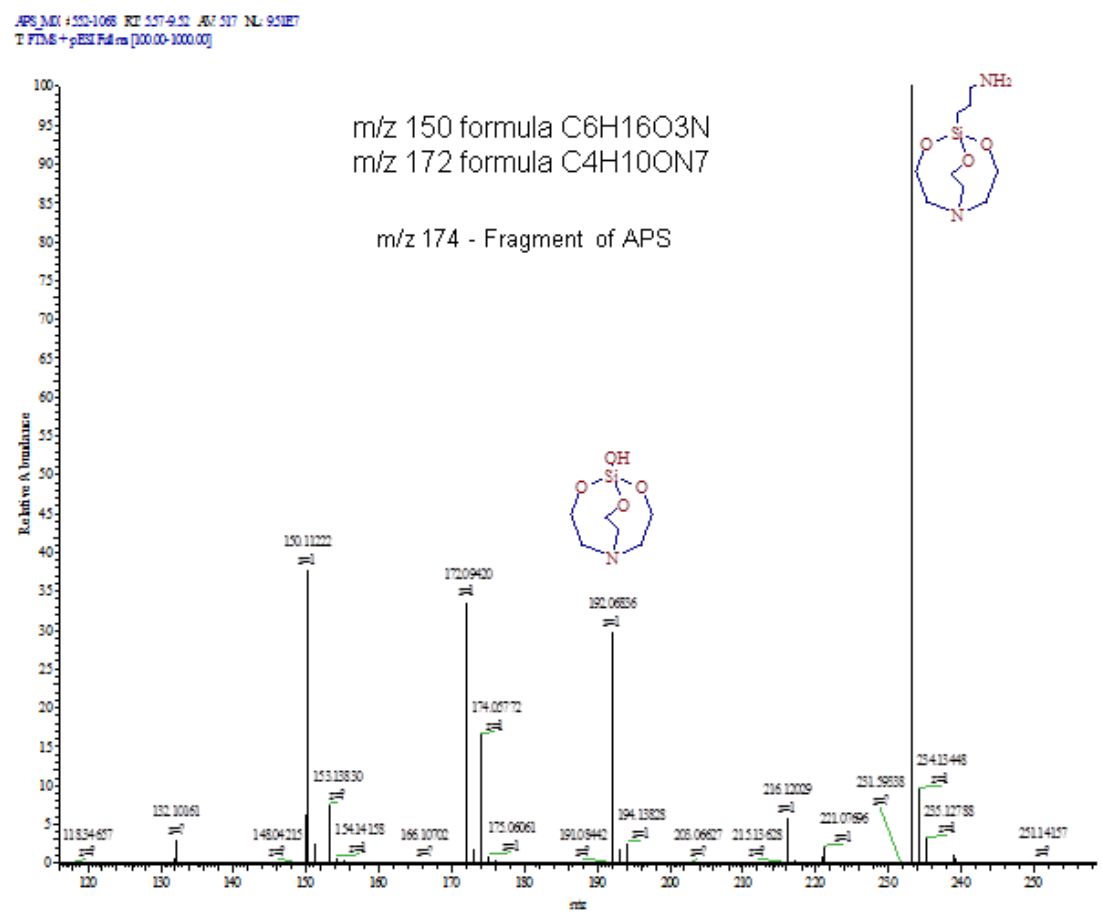

Figure 2. MS-ESi data of purified APS derivative. Presence of APS molecular ion as well as other mass spectrum fragments - the main are illustrated inside the MS spectra.

\section{MALDi-TOF of APS}

Mass spectrometry based on the ionization intermediated by the matrix material (MALDi-TOF) is a powerful method for analysis of polymeric materials, i.e. material of higher molecular weights. Based on the roughness measurements of APS (non-purified) modified mica, the supposed presence of byproducts in the APS reaction mixture could be seen. MALDi-TOF spectrometry was used to prove the presence of high-molecular compounds in the unpurified reaction mixture.
The mass spectra (see Figure 3) indicated that in the crude APS product was found basic molecular ion peak of $\mathrm{m} / \mathrm{z} 233.16$, however also peaks of ions formed from oligo and polymeric products, typically in the $\mathrm{m} / \mathrm{z}$ range of 700 to 1400 . The molecular peaks corresponding to ionization of the matrix are easily identifiable and are shown as the dashed lines in the mass spectra.

The higher molecular peaks are not presented in the MALDi-TOF mass spectra measured after the toluene recrystallization of the APS crude product. 
For the MALDi-TOF mass spectra see Figure 3. This fact together with the reduced surface roughness (APS-modified mica) clearly

demonstrates the suitability and necessity of a two-step purification of APS.

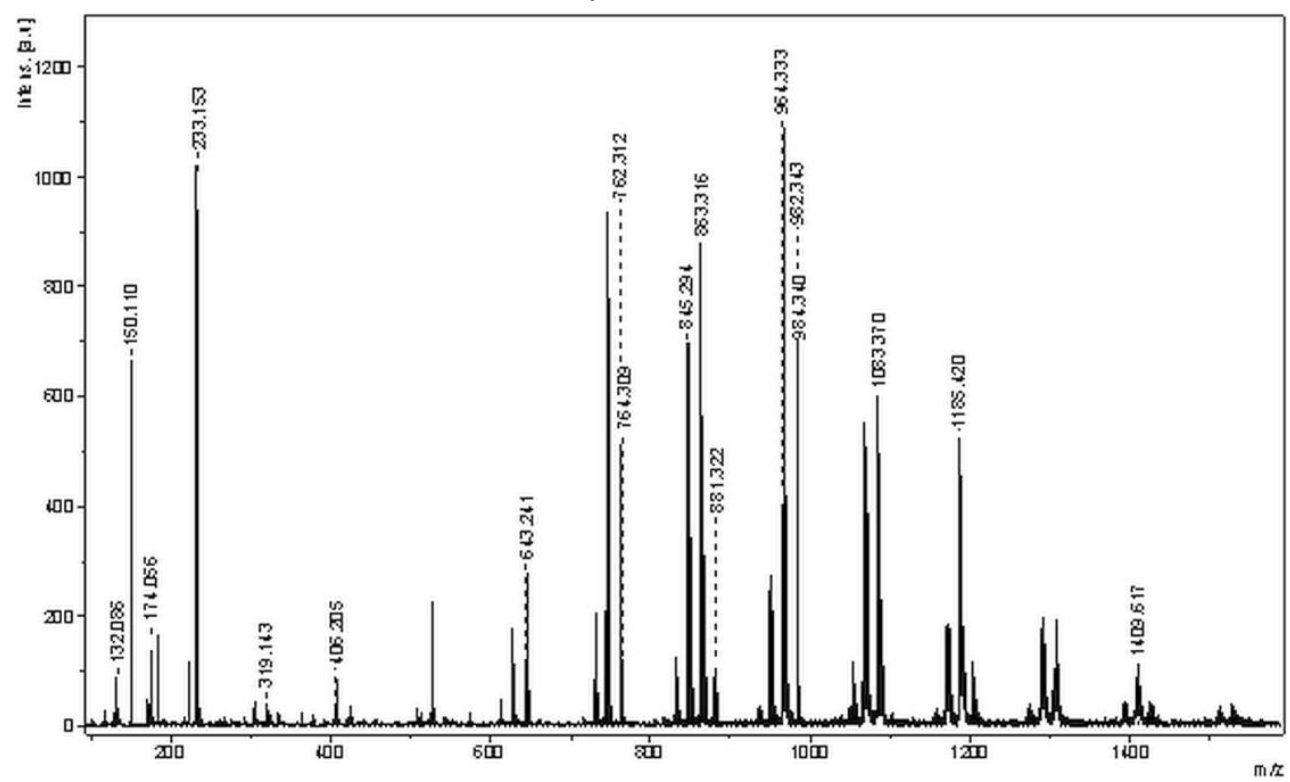

Figure 3. MALDi-TOF spectra of non-purified APS derivatives, presence of specific molecular ions ( $\mathrm{m} / \mathrm{z}$ 233.2), however also molecular ions of side products of APS synthesis are clearly visible. The dashed lines of peaks belong to MALDi matrix (HCCA).

\section{Roughness analysis}

Low roughness of silanized mica surface is an essential condition for suitable DNA surface imaging using AFM microscopy. This microscopy is known to be able to visualize individual biomolecules, including their subunit structure ${ }^{31-33}$. The presence of interfering structures on the surface can significantly affect the force imaging, and therefore it is important to use proper immobilization methods that cannot allow the formation of undesirable surface structures and thus also increasing the surface roughness.

In order to compare the ability of different APS batches to produce a silanized mica surface of the lowest possible roughness, roughness parameter was analyzed by AFM visualization of $1 \times 1 \mu \mathrm{m}$ area, followed by data post-processing in the evaluation software.
First, the effect of two-step purification on surface roughness was studied by silanization of the mica surface, with selected batches of APS taken either before or after purification. The purification of the APS reduced the roughness of the silanized surface $3.5 \mathrm{X}$ in terms of Rms parameter and almost $4 \mathrm{X}$ in $R a$ parameter of roughness (Table 2). Average value of roughness parameters found for the non-purified APS silanized surface were $0.75(\mathrm{Rms})$ and $0.37(R a)$. The values for purified APS were 0.22 and 0.09 for Rms and Ra, respectively.

Advantages of using the purified APS for the preparation of mica with a low degree of surface roughness shows even more when compared with the roughness of APDMES and APTES modified surface (Table 2). These two chemical compounds are conventional modifying agent to treat the mica prior to immobilization of the DNA molecules ${ }^{26}$.

Table 2. Surface roughness ( $\mathrm{Ra}(\mathrm{Sa})$ and $\mathrm{Rms}(\mathrm{Sq})$ ) APS silanized mica surfaces

\begin{tabular}{|cccccc|}
\hline APS fraction $^{*}$ & $\mathrm{Ra}(\mathrm{Sa})$ & $\mathrm{Rms}(\mathrm{Sq})^{*}$ & APS fraction $^{\#}$ & $\mathrm{Ra}(\mathrm{Sa})$ & $\mathrm{Rms}_{(\mathrm{Sq})}{ }^{\#}$ \\
\hline T03 & 0.075 & 0.131 & $\mathrm{~T} 03$ & 0.248 & 0.321 \\
\hline K01A & 0.168 & 0.266 & K01A & 0.299 & 0.396 \\
\hline K02B & 0.082 & 0.135 & K02B & 0.407 & 0.517 \\
\hline K03A & 0.093 & 0.117 & K03A & 0.347 & 0.443 \\
\hline K04A & 0.077 & 0.485 & K04A & 0.252 & 0.327 \\
\hline K04B & 0.078 & 0.132 & K04B & 0.750 & 3.109 \\
\hline K02C & 0.110 & 0.252 & K02C & 0.277 & 0.364 \\
\hline $\mathbf{A}^{\#}$ & 0.095 & 0.222 & A $^{\#}$ & 0.407 & 0.517 \\
\hline APTES & 0.618 & 0.800 & APDMES & 0.360 & 0.460 \\
\hline
\end{tabular}

purified fractions of APS, ${ }^{\#}$ non-purified fractions of APS 


\section{AFM Imaging of Immobilized DNA}

AFM imaging of DNA molecules immobilized on solid surface presented by mica was used to show the practical examples of significance of the use of APS-modified surfaces. DNA of the $246 \mathrm{bp}$ length was immobilized on APS and APDMES modified mica and visualized by tapping mode AFM.

The resulting images can be seen in the Figure 4, presented after data post-processing by GNU software ${ }^{30}$. The difference in the image background, as presented by the silanized layer can be seen as a higher presence of regions containing
non-DNA structures, i.e. regions formed by impurities - in the samples prepared from either non-purified APS or APDMES. This finding offers one more reasons favouring the use of two-steps APS purification before using its crude form or other silanization reagents such as e.g. APDMES or APTES. Similar findings were observed also by other authors ${ }^{34-37}$, describing presence of clearly non-DNA objects on the surface of silanized mica, however, non-APS silanization was used (mainly APTES based) ${ }^{38-40}$.
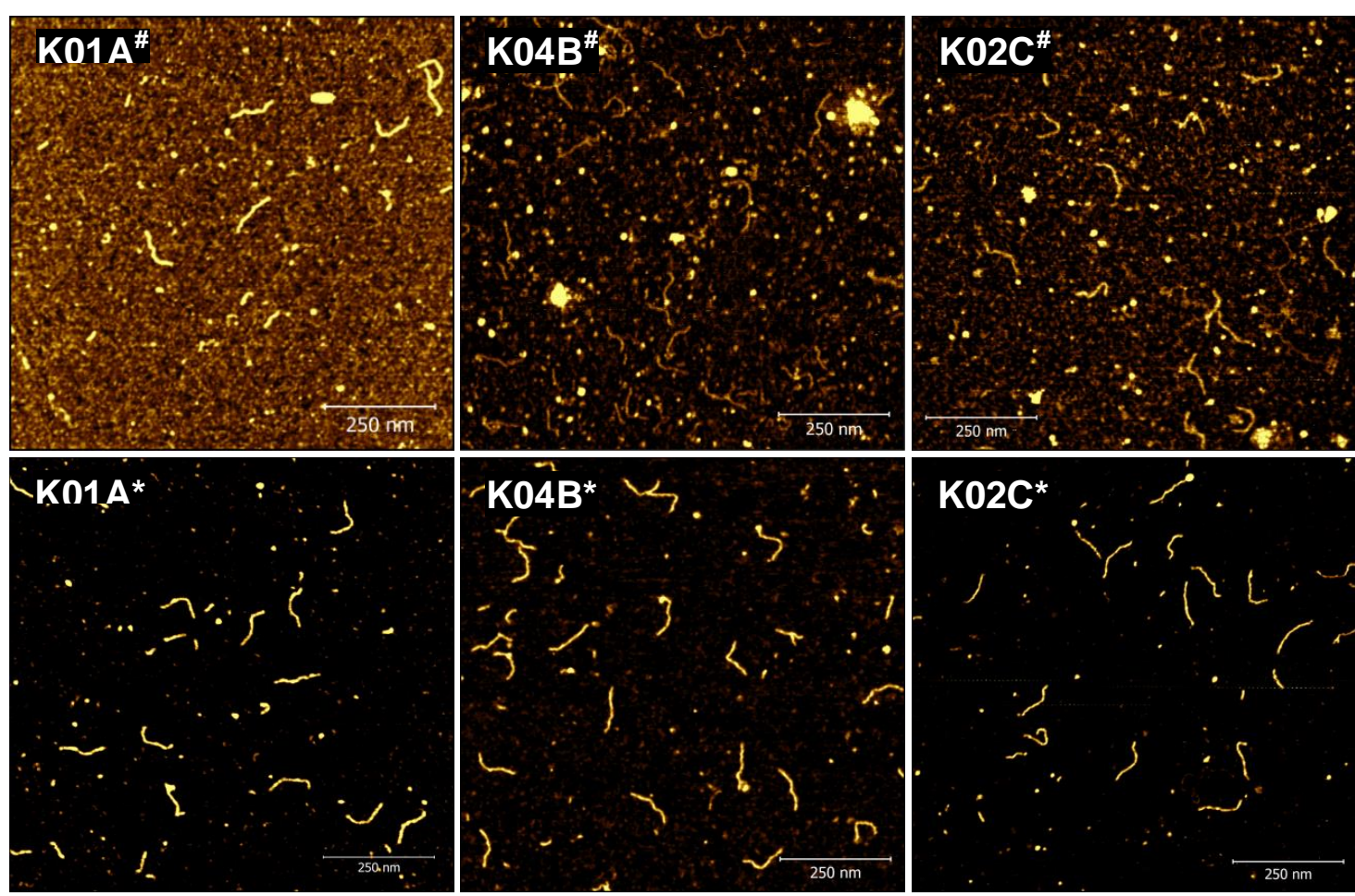

Figure 4. The AFM scans of APS derivative mica with immobilized DNA molecules (246 bp dsDNA). The fractions of APS compound used for mica silanization are inserted in the AFM images as labels.

The */\# sign used means either the purified $(*)$ or non-purified (\#) fraction.

\section{Contact Angle Measurement}

The aging of the APS monolayers formed on the surface of mica was discovered empirically. This finding was obtained after investigation of the DNA structure on the APS-mica surface prepared at different time intervals using AFM scanning. The results of visualization provided varying data both in the density and the shape of the displayed molecules. Effect of changes in the AFM probe and the changes in the DNA structure was eliminated by comparative measurements and standard methods for characterization DNA concentration and structure DNA in the sample. It was found that the only reason may be the aging of silanized layer.

The process of aging was studied by water contact angle measurements during defined intervals with the results shown in the Figure 5. It was found that the aging of the layers is quite a rapid process, changing the water contact angle e.g. from $18^{\circ}$ to $58^{\circ}$ within 48 hours. Whereas PBS modified mica (PBS used instead of APS during modification process), the difference in the water contact angle was from $8^{\circ}$ to $22^{\circ}$ within 48 hours. The largest increase of water contact angle was measured for $6 \mathrm{~W}(1: 1,5)$ and $4 \mathrm{WO}(1,5: 1)$, from $18^{\circ}$ to $58^{\circ}$ and from $20^{\circ}$ to $60^{\circ}$ respectively. This growth of water contact angle value within 48 hours could be explained by various reasons - rearrangement of silanized monolayers ${ }^{41,42}$, surface hydration even in the environment with very low humidity or by a dust adsorption during the sample manipulation. The influence of the latest factors, such as deposition of solid particles from air was minimized by storing the APS modified and control PBS mica sheets inside the desiccator in a clean room. Obviously the only suitable solution is the use of modified mica to immobilize the DNA immediately after the silanization process, or after a constant time interval. Storage of APS-modified mica is therefore not suitable. 

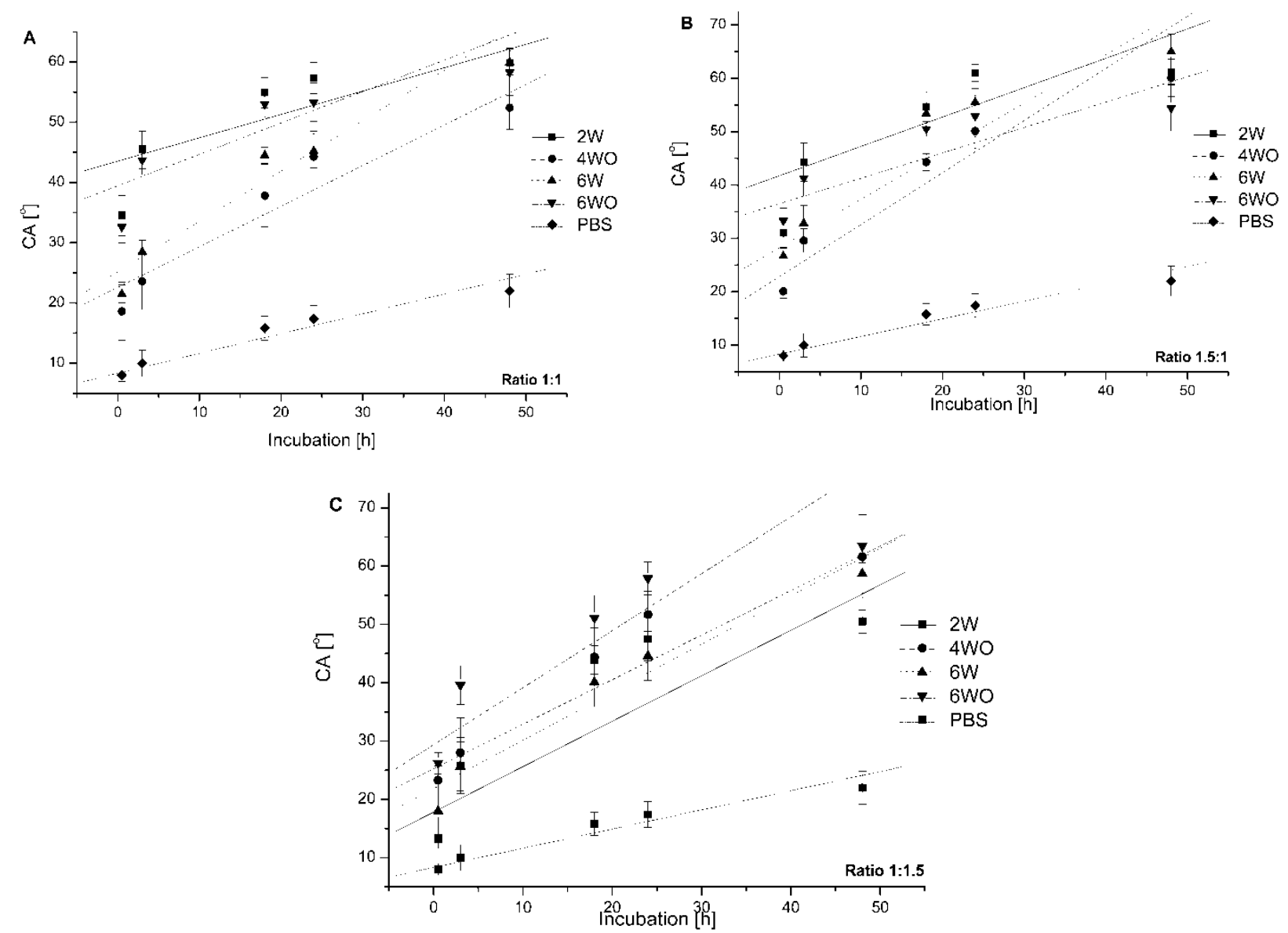

Figure 5. Water contact angle of the mica surface modified with APS derivatives, measured in various time intervals. Effect of different APS types - prepared with following APTMS: TEOA molar ratio 1:1 (graph A)

1.5:1 (graph B) 1:1.5 (graph C) is shown in the graphs.

\section{Conclusion}

Silanization of mica surface is an effective method for immobilization of DNA and other negatively charged molecules for AFM single molecular imaging.

The presented work describes modified way of synthesis of 1-(3-aminopropyl)silatrane (APS) based on a new derivative APTMS ((3-aminopropyl)trimethoxysilane). APS is an innovative, however commercially unavailable, silanization compound allowing mica modification within 10 minutes interval. Various ways and experimental conditions (such as reaction mechanism, various reaction ratios, effect of catalyzer and purification protocol) were studied and optimized. Kinetically driven reaction pathway was found to be optimal, when triethanolamine: aminopropyltrimethoxysilane molar ratio 1:1.5 was used, thus providing a high reaction yield over $80 \%$ comparing to previously published works. However, also necessity of two step purification process composed of vacuum evaporation followed by toluene recrystallization is shown. The mass spectrometry MS-ESi (MS/MS) showed the presence of APS in the final reaction mixture by detection of the primary molecular ion with $\mathrm{m} / \mathrm{z}$ value 231.5. Roughness of APS modified mica surface is an essential criterion for proper imaging of DNA molecules on a single molecular level. This parameter was investigated, when mica surface was modified by purified and crude APS, four times lower roughness of the surface treated with purified APS was found. This effect is probably caused by increased content of oligomers (reaction byproducts) and unreacted triethanolamine in a crude product. These molecules are eliminated by recrystallization process; as it was confirmed by MALDi-TOF spectrometry, showing decreased presence of various oligomeric compounds in purified APS (molecular ions of $\mathrm{m} / z$ from 700 to 1400). AFM imaging of dsDNA (246 bp length) molecules immobilized on modified mica surfaces illustrates practical use of the APS compound as silanization compound.

\section{References}

1- E. C. Cesconetto, F. S. A. Junior, F. a. P. Crisafuli, O. N. Mesquita, E. B. Ramos, and M. S. Rocha, Phys. Chem. Chem. Phys., 2013, 15, 11070. 
2- V. Cassina, D. Seruggia, G. L. Beretta, D. Salerno, D. Brogioli, S. Manzini, F. Zunino, and F. Mantegazza, Eur. Biophys. J. EBJ, 2011, 40, 59.

3- V. Cassina, M. Manghi, D. Salerno, A. Tempestini, V. Iadarola, L. Nardo, S. Brioschi, and F. Mantegazza, Biochim. Biophys. Acta BBA - Gen. Subj., 2016, 1860, 1.

4- J. Yang, K. Takeyasu, and Z. Shao, FEBS Lett., 1992, 301, 173.

5- P. Wagner, FEBS Lett., 1998, 430, 112.

6- J. Adamcik, D. V. Klinov, G. Witz, S. K. Sekatskii, and G. Dietler, FEBS Lett., 2006, 580, 5671.

7- N. Borovok, T. Molotsky, J. Ghabboun, H. Cohen, D. Porath, and A. Kotlyar, FEBS Lett., 2007, 581, 5843.

8- J. Mou, D. M. Czajkowsky, Y. Zhang, and Z. Shao, FEBS Lett., 1995, 371, 279.

9- V. Horňáková, J. Přibyl, and P. Skládal, Monatshefte Für Chem. - Chem. Mon., 2016, 147, 865 .

10- A. Podestà, L. Imperadori, W. Colnaghi, L. Finzi, P. Milani, and D. Dunlap, J. Microsc., 2004, 215, 236.

11- P. A. Wiggins, T. van der Heijden, F. MorenoHerrero, A. Spakowitz, R. Phillips, J. Widom, C. Dekker, and P. C. Nelson, Nat. Nanotechnol., 2006, $1,137$.

12- N. Liu, T. Bu, Y. Song, W. Zhang, J. Li, W. Zhang, J. Shen, and H. Li, Langmuir, 2010, 26, 9491.

13- F. Zhang, P. Zhang, J. Hou, X. Yun, W. Li, Q. $\mathrm{Du}$, and Y. Chen, ACS Appl. Mater. Interfaces, 2015, 7, 8699 .

14- M. V. Maslova, L. G. Gerasimova, and W. Forsling, Colloid J., 2004, 66, 322.

15- N. C. Santos and M. A. R. B. Castanho, Biophys. Chem., 2004, 107, 133.

16- J. Vesenka, M. Guthold, C. L. Tang, D. Keller, E. Delaine, and C. Bustamante, Ultramicroscopy, 1992, 42-44 ( Pt B), 1243.

17- Y. Lyubchenko, L. Shlyakhtenko, R. Harrington, P. Oden, and S. Lindsay, Proc. Natl. Acad. Sci. U. S. A., 1993, 90, 2137.

18- Y. L. Lyubchenko and L. S. Shlyakhtenko, Proc. Natl. Acad. Sci., 1997, 94, 496.

19- R. G. Acres, A. V. Ellis, J. Alvino, C. E. Lenahan, D. A. Khodakov, G. F. Metha, and G. G. Andersson, J. Phys. Chem. C, 2012, 116, 6289.

20- C. P. Tripp and M. L. Hair, Langmuir, 1995, 11, 1215.

21- "Spectroscopic Analysis of Heterogeneous Catalysts, Part A: Methods of Surface Analysis", ed. Morrow, B. and Fierro L.G., 1990, Elsevier, Amsterdam.

22- C. P. Tripp and M. L. Hair, Langmuir, 1991, 7, 923.

23- L. D. White and C. P. Tripp, J. Colloid Interface Sci., 2000, 224, 417.

24- W. J. Tiner Sr, V. N. Potaman, R. R. Sinden, and Y. L. Lyubchenko, J. Mol. Biol., 2001, 314, 353.

25- Voronkov M.G., Fedorova E.O., and Chernov N.F., RussJ Org Chem, 1994, 30.

26- L. S. Shlyakhtenko, A. A. Gall, A. Filonov, Z. Cerovac, A. Lushnikov, and Y. L. Lyubchenko, Ultramicroscopy, 2003, 97, 279.

27- A.-C. Dumitriu, M. Cazacu, S. Shova, C. Turta, and B. C. Simionescu, Polyhedron, 2012, 33, 119.

28- N. Crampton, W. A. Bonass, J. Kirkham, and N. H. Thomson, Ultramicroscopy, 2006, 106, 765.

29- Y. L. Lyubchenko and L. S. Shlyakhtenko, Methods, 2009, 47, 206.

30- D. Nečas and P. Klapetek, Open Phys., 2012, 10, 181.

31- P.-E. Milhiet, F. Gubellini, A. Berquand, P. Dosset, J.-L. Rigaud, C. Le Grimellec, and D. Lévy, Biophys. J., 2006, 91, 3268.

32- Z. Shao, News Physiol. Sci. Int. J. Physiol. Prod. Jointly Int. Union Physiol. Sci. Am. Physiol. Soc., 1999, 14, 142.

33- A. San Paulo and R. García, Biophys. J., 2000, $78,1599$.

34- A. Japaridze, D. Vobornik, E. Lipiec, A. Cerreta, J. Szczerbinski, R. Zenobi, and G. Dietler, Macromolecules, 2016, 49, 643.

35- H. Labit, A. Goldar, G. Guilbaud, C. Douarche, O. Hyrien, and K. Marheineke, BioTechniques, 2008, 45, 649.

36- L. H. Pope, M. C. Davies, C. A. Laughton, C. J. Roberts, S. J. Tendler, and P. M. Williams, $J$. Microsc., 2000, 199, 68.

37- N. Crampton, W. A. Bonass, J. Kirkham, and N. H. Thomson, Langmuir, 2005, 21, 7884.

38- W. Wang and M. W. Vaughn, Scanning, 2008, 30, 65.

39- Y. L. Lyubchenko, A. A. Gall, and L. S. Shlyakhtenko, Methods Mol. Biol. Clifton NJ, 2014, 1117, 367.

40- M. Mahut, M. Leitner, A. Ebner, M. Lämmerhofer, P. Hinterdorfer, and W. Lindner, Anal. Bioanal. Chem., 2011, 402, 373.

41- J. K. Puri, R. Singh, and V. K. Chahal, Chem. Soc. Rev., 2011, 40, 1791.

42- I. Kovács, E. Matern, E. Sattler, C. E. Anson, and L. Párkányi, J. Organomet. Chem., 2009, 694, 14. 